Original Paper http://ajol.info/index.php/ijbcs http://indexmedicus.afro.who.int

\title{
Assainissement de l'environnement par la valorisation des ressources non conventionnelles en alimentation de porcs en croissance
}

\author{
Carine Christiane HEDJI ${ }^{1 *}$, Marcel HOUINATO ${ }^{2}$, Frédéric HOUNDONOUGBO ${ }^{3}$ et \\ Emile FIOGBE ${ }^{1}$
}
${ }^{1}$ Laboratoire de Recherche sur les Zones Humides (LRZH), Département de Zoologie, Faculté des Sciences et Techniques (FAST), Université d'Abomey-Calavi (UAC), République du Bénin.
${ }^{2}$ Laboratoire de Zootechnie, Département de Production Animale, Faculté des Sciences Agronomiques (FSA)/ Université d'Abomey-Calavi (UAC), République du Bénin.
${ }^{3}$ Laboratoire de Recherche Avicole et de Zoo-Economie, Département de Production Animale Faculté des Sciences Agronomiques/ Université d'Abomey-Calavi, 01 BP 526 Cotonou, République du Bénin.
*Auteur correspondant, E-mail : christmel13@yahoo.fr, Tel :(0022997 1376 13)

\section{RESUME}

La présente étude s'est intéressée à la comparaison des performances zootechniques et économiques des porcs nourris avec des aliments commerciaux et des aliments non conventionnels. Au total, 90 porcelets métis (race locale X Large white) âgés de 60 à 90 jours dont 45 mâles castrés et 45 femelles, ont été répartis en 15 lots de 6 animaux, à raison de 3 loges de 6 porcelets par traitement alimentaire. Chaque lot a été nourri à volonté avec un aliment commercial et quatre aliments contenant différents taux de Azolla, Moringa, son de riz, viscères de poulet Cobb 500 et de poisson Oreochromis niloticus. Une différence significative $(\mathrm{P}<0,05)$ a été notée entre les gains moyens quotidiens (GMQ). Après 90 jours d'expérimentation, les GMQ étaient de $197 \mathrm{~g} / \mathrm{j}$ pour les porcs du lot à aliment commercial et de 63 à 101g/j pour ceux soumis aux aliments à base de ressources non conventionnelles. Il ressort des calculs économiques que le coût alimentaire (Ca) par kg de gain de poids (GP) était plus faible pour l'aliment non conventionnel (716 FCFA/kg GP) par rapport à l'aliment commercial (830 FCFA/kg GP). Il est donc possible d'utiliser ces ressources non conventionnelles pour nourrir de façon efficiente des porcs dans un système intégré d'exploitations familiales tout en rendant l'environnement sain.

(C) 2015 International Formulae Group. All rights reserved.

Mots clés : Aliment, performance, porcs, rentabilité économique.

\section{Sanitation of the environment by the valorisation of non-conventional resources in feed of growing pigs}

\section{ABSTRACT}

The present study was focused on the comparison of zootechnic and economic performance of pigs nourished with commercial and non-conventional feed. In total, 90 crossbred piglets (Landrace x Large white) 
aged 60 to 90 days with 45 castrated males and 45 females, were divided into 15 groups of 6 animals at the rate of 3 lodges of 6 piglets per treatment. Each batch was fed ad libitum with commercial feed and four feed containing different levels of Azolla, Moringa, rice bran, chicken Cobb 500 and fish Oreochromis niloticus viscera. A significant difference $(\mathrm{P}<0.05)$ was observed between Average Daily Gain (ADG). After 90 days of experimentation, the ADG were $197 \mathrm{~g} /$ day for pigs nourished with commercial feed and 63 to $101 \mathrm{~g} /$ day for those subjected to feed made from unconventional resources. It emerges from the economic calculations that the feed cost (Ca) per kg of Weight Gain (WG) was lower for non-conventional feed (716 FCFA / kg WG) than the commercial feed one ( 830 FCFA / kg WG). It is therefore possible to use these unconventional resources efficiently to feed pigs in an integrated system of family farms while making healthy environment.

(C) 2015 International Formulae Group. All rights reserved.

Keywords: Feed, performance, pigs, economic profitability.

\section{INTRODUCTION}

L'assainissement durable peut, grâce à des innovations, revêtir un caractère productif par la valorisation en élevage des éléments nutritifs contenus dans les ressources alimentaires locales. L'alimentation est l'un des facteurs les plus importants à maitriser pour le développement de l'élevage sous les tropiques en général et au Bénin en particulier. Les charges liées à l'alimentation représentent $70 \%$ des charges totales de production. La réduction des coûts alimentaires devient une préoccupation importante pour les petits éleveurs des pays chauds. Dans les élevages en général et au Bénin, la quasi-totalité des matières premières utilisées sont des ressources alimentaires classiques comme les tourteaux de soja, d'arachide, de palmiste ou farine de poisson ; les acides aminés synthétiques et les compléments minéralo-vitaminés qui sont pour la plupart importés, chers et parfois difficiles à trouver (Aboh et al., 2002 ; Braine, 2008). L'utilisation des aliments locaux non conventionnels dans l'alimentation des animaux apparaît raisonnablement comme une alternative aux aliments commerciaux classiques (Aboh et al., 2002). Parmi ceux-ci, le Moringa occupe une place de choix, du point de vue de son impact potentiel sur l'augmentation de la productivité (Fuglie, 2001). Les sous-produits locaux issus de la transformation du riz, notamment les sons, non consommés par l'homme, sont valorisés par les animaux dont les porcs (Mopaté,
2008). De plus, baser l'élevage des animaux sur les ressources locales permettrait d'augmenter la productivité par unité de ressources fondamentales disponibles. Du fait de ses caractéristiques nutritionnelles, l'Azolla est convenable pour la consommation humaine et pour des suppléments alimentaires chez divers animaux comme: poisson, canards, bétail, volaille, etc. afin de réduire le coût de l'alimentation (Hassan et al., 2009 ; Raja et al., 2012). Les co-produits d'abattoir (sang, poumons, intestins) de même que ceux de la pêche (têtes, viscères, arêtes, queues, nageoires, déchets d'usines), sont insuffisamment récupérés et valorisés car ils constituent d'excellents compléments protéiques (Ovissipour et al., 2009). Le traitement des sous-produits de la volaille dans l'alimentation animale est un bon moyen pour atténuer les problèmes environnementaux causés par la transformation de la volaille. Ces sousproduits peuvent libérer dans l'environnement des bactéries et des virus à partir des vecteurs comme les insectes, la vermine, qui entraînent la contamination de l'eau (lessivage des nutriments et micro-organismes pathogènes) et la pollution de l'air (gaz nocifs et odeurs nuisantes). Cette étude vise donc à valoriser le son de riz, l'Azolla, le Moringa et les viscères de poulet Cobb 500 et ou de poisson Oreochromis niloticus dans l'alimentation des porcs pour une agriculture familiale intégrée. 


\section{MATERIEL ET METHODES Dispositif expérimental}

Quatre-vingt-dix porcelets croisés Local x Large white (45 mâles castrés et 45 femelles) âgés de 2 à 3 mois et pesant initialement $9,5 \pm 0,18 \mathrm{~kg}$ ont été répartis au hasard dans 15 loges expérimentales, de dimensions $1,5 \mathrm{~m} \times 3 \mathrm{~m}$, construites en bois et au sol cimenté, dans un dispositif complètement randomisé sur une durée de 90 jours. Chaque loge était munie d'un abreuvoir rectangulaire et d'une mangeoire linéaire de dimension $0,5 \mathrm{~m} \times 0,5 \mathrm{~m} \times 0,3 \mathrm{~m}$. Les porcelets ont été soumis à quatre aliments expérimentaux et un témoin avec trois répétitions par traitement après 14 jours d'adaptation. La composition des aliments d'élevage est présentée dans le Tableau 1.

Les pesées ont été hebdomadaires. Les consommations d'aliments ont été évaluées quotidiennement. L'évaluation des performances zootechniques a été faite sur la base de l'ingestion alimentaire (IA), le gain moyen quotidien (GMQ), l'indice de consommation (IC) et le coût alimentaire de production du kilogramme de gain de poids. En se basant d'une part, sur le coût de la main d'œuvre pour la récolte et le séchage de Azolla et de Moringa, et d'autre part, sur les charges de transport, la valeur économique des feuilles séchées a été estimée à 125 et 100 FCFA par $\mathrm{kg}$ respectivement pour le Moringa et l'Azolla . Les farines de viscères de poulet et de poisson ont été estimées respectivement à 300 et 250 FCFA. Sur le marché local, le coût du son de riz est $100 \mathrm{FCFA} / \mathrm{kg}$ et celui de l'aliment composé commercial est 180 FCFA/kg.

\section{Analyses statistiques}

Les données collectées ont été analysées dans le logiciel SAS version 9.4. Afin de tester l'effet du traitement alimentaire sur les différents paramètres zootechniques, une analyse de la variance (ANOVA, un facteur) a été réalisée avec la procédure de General Linear Model (GLM) selon le model suivant :
$\mathrm{Y}_{\mathrm{i}}=\mu+\mathrm{T}_{\mathrm{i}}+\varepsilon_{\mathrm{i}}$ avec $\mathrm{Y}_{\mathrm{i}}=$ Observations de la des variable dépendante, $\mu=$ moyenne générale, $\mathrm{T}_{\mathrm{i}}=$ Effet du traitement alimentaire, $\varepsilon_{\mathrm{i}}=$ Erreur résiduelle.

Par ailleurs le test de Student-NewmanKeuls a été réalisé afin d'identifier les traitements homogènes (n'ayant pas d'effets différentiels les uns par rapport aux autres) aux regards du comportement des paramètres. L'effet du traitement alimentaire est dit significatif si $\mathrm{P}<0,05$.

\section{RESULTATS}

L'ingestion d'aliment par les porcs n'a pas été similaire $(\mathrm{p}<0,05)$ pour les cinq groupes de traitements alimentaires A0, A1, $\mathrm{A} 2$, A3 et A4 (Figure 1). Les traitements alimentaires à base de ressources non conventionnelles ont significativement $(\mathrm{p}<$ $0,05)$ affecté les paramètres zootechniques des porcs. La croissance pondérale a été irrégulière au cours de l'essai. Les porcs du lot témoin ont eu la meilleure croissance (Figure 2).

$\mathrm{Au}$ terme de l'expérimentation, les porcs de poids initial $9,5 \mathrm{~kg}$ ont pesé 34,17 , 19,20 et $22 \mathrm{~kg}$ respectivement pour les traitements alimentaires de $\mathrm{A} 0$ à $\mathrm{A} 4$. Le gain moyen quotidien (GMQ) a été significativement différent entre les 5 traitements. Le GMQ le plus élevé a été enregistré au niveau du lot témoin. Ce GMQ vaut 1,95 à 3,12 fois ceux obtenus dans les traitements à base des ressources alimentaires non conventionnelles. L'indice de consommation et le coût de l'alimentation par $\mathrm{kg}$ de gain de poids vif corporel ont été significativement affectés par les traitements alimentaires $(\mathrm{P}<0,05)$ comparativement au témoin A0. Le meilleur indice de consommation a été enregistré au niveau du traitement témoin et le meilleur coût de l'alimentation a été celui du traitement A4 enrichi aux viscères de poulet (Tableau 2). Le traitement alimentaire A4 a donné les meilleures performances au niveau des aliments non conventionnels utilisés. 


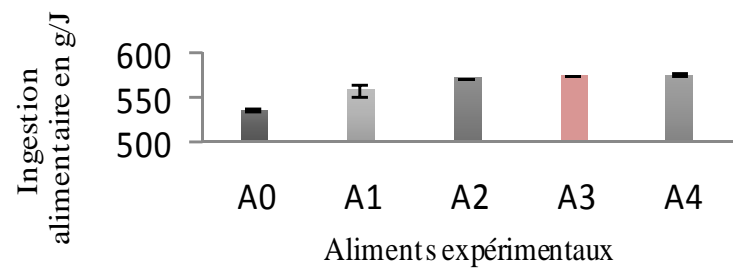

Figure 1 : Consommation alimentaire en fonction des traitements.

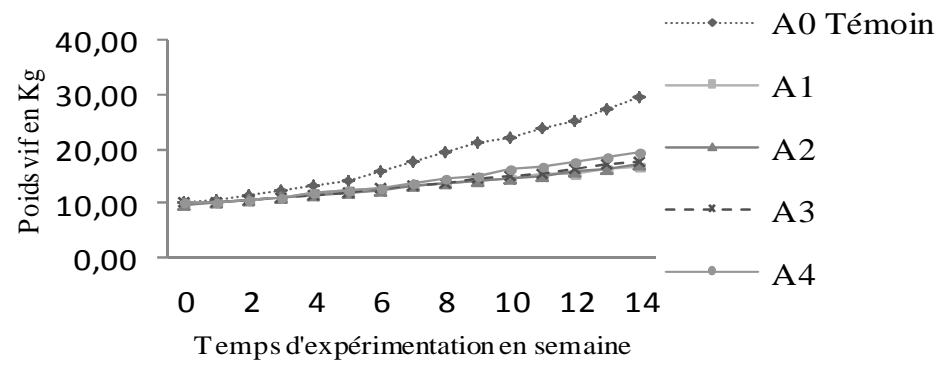

Figure 2 : Evolution du poids pondéral en fonctions des traitements alimentaires.

Tableau 1 : Ingrédients et composition nutritionnelle des aliments expérimentaux.

\begin{tabular}{|c|c|c|c|c|c|}
\hline \multirow{2}{*}{ Ingrédients (\%) } & \multicolumn{5}{|c|}{ Aliments expérimentaux } \\
\hline & A0 & A1 & A2 & A3 & A4 \\
\hline Maïs & 29 & & & & \\
\hline Son de blé & 24,65 & & & & \\
\hline Tourteau palmiste & 32,5 & & & & \\
\hline Tourteau de coton & 5 & & & & \\
\hline Farine de poisson & 5 & & & & \\
\hline Coquille & 1,5 & & & & \\
\hline $\mathrm{C} 5$ & 1,5 & & & & \\
\hline Phosphate & 0,5 & & & & \\
\hline Sulfate & 0,05 & & & & \\
\hline Sel & 0,3 & & & & \\
\hline Azolla & & 40 & 40 & 40 & 40 \\
\hline Moringa & & 25 & 25 & 25 & 25 \\
\hline Son de riz & & 30 & 30 & 30 & 30 \\
\hline Viscères de poulet & & 0 & 1,5 & 3,5 & 5 \\
\hline Viscères de poisson & & 5 & 3,5 & 1,5 & 0 \\
\hline Total & 100 & 100 & 100 & 100 & 100 \\
\hline \multicolumn{6}{|l|}{ Composition nutritionnelle } \\
\hline Matière sèche $(\%)$ & 94 & 86 & 85 & 85 & 85 \\
\hline Teneur en protéine $(\%)$ & 19,8 & 18,7 & 18 & 19,59 & 19,95 \\
\hline Teneur en lipide $(\%)$ & 9,71 & 5,3 & 5,56 & 5,89 & 6,16 \\
\hline Teneur en fibres $(\%)$ & 17.31 & 18.41 & 18.41 & 18.41 & 18.41 \\
\hline Energie métabolisable (Kcal/Kg MS) & 2604 & 2211 & 2152 & 2283 & 2314 \\
\hline Prix du kg d'aliment (F CFA) & 180,1 & 113 & 114,5 & 115,5 & 116,3 \\
\hline
\end{tabular}


Tableau 2: Influence des traitements alimentaires sur les paramètres zoo-économiques.

\begin{tabular}{|c|c|c|c|c|c|c|}
\hline Paramètres & A0 & A1 & A2 & A3 & A4 & $\mathbf{P}$ \\
\hline Poids initial (Kg) & $10 \pm 0,01^{\mathrm{a}}$ & $9,99 \pm 0,06^{\mathrm{a}}$ & $9,9 \pm 0,06^{\mathrm{a}}$ & $9,98 \pm 0,01^{\mathrm{a}}$ & $9,96 \pm 0,04^{\mathrm{a}}$ & $>0.05$ \\
\hline Poids final $(\mathrm{Kg})$ & $34,58 \pm 0,08^{a}$ & $17,12 \pm 0,8^{\mathrm{e}}$ & $19,27 \pm 0,23^{d}$ & $20,29 \pm 0,2^{c}$ & $22,58 \pm 0,25^{b}$ & $<0.0001$ \\
\hline GMQ (g) & $197,74 \pm 0,5^{\mathrm{a}}$ & $63 \pm 4,04^{\mathrm{e}}$ & $75,13 \pm 2^{d}$ & $80,69 \pm 0,93^{\mathrm{c}}$ & $101,4 \pm 1,14^{\mathrm{d}}$ & $<0.0001$ \\
\hline Indice de consommation (IC) & $4,61 \pm 0,01^{\mathrm{d}}$ & $11,13 \pm 0,87^{\mathrm{a}}$ & $10,68 \pm 0,29^{\mathrm{a}, \mathrm{b}}$ & $9,99 \pm 0,13^{b}$ & $7,95 \pm 0,11^{\mathrm{c}}$ & $<0.0001$ \\
\hline Coût alimentaire FCFA & $830 \pm 1,47^{\mathrm{d}}$ & $1081 \pm 32,16^{\mathrm{a}}$ & $942,61 \pm 25,72^{b}$ & $891,67 \pm 0,93^{\mathrm{c}}$ & $716,06 \pm 10,16^{\mathrm{e}}$ & $<0.0001$ \\
\hline
\end{tabular}

Les valeurs se trouvant sur la même ligne et partageant la même lettre ne sont pas significativement différentes.

\section{DISCUSSION}

Les cinq aliments expérimentaux sont presque iso-protéiques, mais ils contiennent différents niveaux d'énergies métabolisables, de matières grasses et de fibres brutes. Les compositions chimiques faites des rations expérimentales ont montré des valeurs différentes pour les matières grasses et les fibres brutes. La quantité d'ingestion alimentaire (IA) notée chez les porcs nourris avec les cinq rations indique une bonne appétence des cinq aliments. Ces résultats sont conformes à ceux obtenus chez les porcs locaux au Bénin et au Burkina (Codjo, 2003 ; Bougouma et al., 2005) qui étaient de 523 à $754 \mathrm{~g} / \mathrm{j}$. Dans des régimes riches en fibres, les animaux accroissent la consommation alimentaire pour compenser partiellement la baisse de la concentration énergétique (Bougouma et al., 2005). Le prix du kilogramme de l'aliment témoin est de 180 FCFA contre $113,114,115$ et 116 FCFA $/ \mathrm{kg}$ respectivement pour les aliments $\mathrm{A} 1, \mathrm{~A} 2, \mathrm{~A} 3$ et A4. La valorisation de ressources non conventionnelles a réduit le prix de l'aliment.

Les performances et le développement de l'animal dépendent principalement de l'ingestion alimentaire de l'animal (Saxena, 2011). En général, les performances de croissance ont été meilleures dans le lot témoin que dans les autres lots. Le gain moyen quotidien des animaux a été généralement faible, passant de $101 \mathrm{~g}$ à $63 \mathrm{~g}$ pour les rations contenant $100 \%$ de ressources non conventionnelles, alors que celui du témoin est de $197 \mathrm{~g}$. Ces résultats sont en deçà de ceux de Saikia et Bhar, 2010; Adesehinwa, 2007 ; Adesehinwa et al., 2011 qui ont utilisé respectivement les déchets de cuisine, le tourteau de palmiste et les épluchures de manioc d'alimentation des porcs. Cette différence des GMQ a été notée par Ezekwe et al. (2011) et Agbabiaka et al. (2014) qui ont travaillé sur les porcs croisés Large White et Landrace. Il ressort de ce constat que la croissance du porc dépend de la qualité de l'aliment et du potentiel génétique de l'animal. Le GMQ des lots soumis aux aliments non conventionnels est comparable aux résultats de Youssao et al. (2004) qui ont mené des essais sur des porcs locaux en claustration. Le faible GMQ de ces lots au bout de 90 jours s'expliquerait entre autre par les teneurs élevées de celluloses brutes mais aussi par le faible niveau d'énergie des aliments. Les sous-produits artisanaux et agroindustriels riches en fibres comme le son de riz ont une faible concentration énergétique par rapport aux besoins des porcs en engraissement (Meffeja et al., 2006). D'autres études ont confirmé l'effet négatif des niveaux élevés de fibres alimentaires sur la digestibilité de certains nutriments (Zanfi et Spanghero, 2012 ; Zanfi et al., 2014). Wang et al. (2006) ont rapporté une diminution de la digestibilité iléale apparente et fécale des acides aminés y compris les aliments fibreux dans les régimes alimentaires pour les porcs en croissance. De plus, Wang et al. (2004) ont constaté que la supplémentation du régime alimentaire des porcs en croissance avec de la pâte de son de blé et la betterave à sucre réduit la digestibilité fécale de l'énergie. Mais une haute teneur en fibres dans l'alimentation des porcs conduit à la production de viande maigre plus apprécié dans le cas du porc par les consommateurs asiatiques et africains (Patricot 2002; Mopaté et Kaboré-Zoungrana, 2005). Le traitement témoin avait une teneur plus élevée en matières grasses. L'extrait 
éthéré a un pouvoir calorifique élevé. Cela pourrait expliquer la différence significative entre les gains moyens quotidiens des différents groupes de porcs. En effet, l'un des principaux composants de la régulation de la prise alimentaire est l'énergie digestible (ED) (Gidenne et Lebas, 2005). L'augmentation de l'énergie dans l'aliment témoin a été donc induite par la teneur en matières grasses qui est en forte proportion dans cet aliment commercial. L'indice de consommation $(4,61)$ a été meilleur chez les porcs de la ration témoin. Il est 1,15 fois supérieur à celui du lot témoin de Adesehinwa (2009). Les valeurs élevées des IC (7 à 11) dans les aliments à ressources non conventionnelles (A1 à $\mathrm{A} 4)$ indiqueraient que les porcs ont bien consommé sans mieux valoriser ces aliments. Ces résultats sont largement au-dessus de ceux trouvés par Adesehinwa (2009); Dube et al. (2011); Ezekwe et al. (2011) et Agbabiaka et al. (2014) sur des porcs croisés (Large White et Landrace).

Le coût des aliments par kg de gain de poids vif des porcs a été relativement bas au niveau de l'aliment A4 (716 FCFA) par rapport à $\mathrm{A} 0$ (830 FCFA). La réduction du coût global de l'alimentation a également été rapportée par diverses études (Ezekwe et al., 2011 ; Agbabiaka et al., 2014; Zanfi et al., 2014). Le rendement économique est essentiellement dû à la réduction du coût des ressources non conventionnelles et locales. L'aliment local est efficace en raison de son abondance et de la collecte facile. Les ressources alimentaires non conventionnelles n'ont pas permis de réaliser de très bonnes performances à cause du taux de fibres lié à la teneur élevée surtout du son de riz dans l'aliment. Mais, cette étude a montré qu'il est possible de valoriser ces ressources alimentaires non conventionnelles dans l'alimentation des porcs en croissance pour une agriculture familiale. Ces aliments peuvent être améliorés par la substitution partielle du son de riz par l'ajout de tourteau de palmiste et de son de maïs et autres ressources locales disponibles. Cette valorisation permettrait aussi la résolution des problèmes environnementaux et la création de la valeur ajoutée.

\section{Conclusion}

Il ressort de cette étude que la valorisation de nos ressources non conventionnelles est possible dans l'alimentation des porcs. Cette valorisation participera à une meilleure gestion de l'environnement. Les aliments utilisés (Azolla, Moringa, son de riz, viscères de poulet et/ ou de poisson) n'ont pas donné de performances meilleures par rapport aux aliments commerciaux conventionnels. $\mathrm{Ce}$ régime composé uniquement de ressources non conventionnelles peut être amélioré par l'ajout d'autres ingrédients comme le son de maïs, le tourteau de palmiste, les épluchures de manioc, d'igname et de patate, etc. La croissance pondérale des porcs par les quatre aliments non conventionnels appliqués n'est pas négligeable. L'utilisation de la formule alimentaire A4 (son de riz, Moringa, Azolla, viscères de poulet) est indiquée dans une agriculture familiale ou dans un système d'intégration agriculture - élevage. Elle participera à la réduction du coût de l'alimentation et à l'assainissement de l'environnement.

\section{REMERCIEMENTS}

Cette étude ne pourra pas être effectuée sans le soutien financier du projet OPASISI des Fonds Compétitifs de l'Université d'Abomey-Calavi. Les auteurs remercient Dr Adjolohoun Sébastien, Dr Adénilé Adam et Mr Effio Apollinaire pour leur contribution

\section{REFERENCES}

Aboh AB, Olaafa M, Dossou-Gbété GSO, Dossa AD, Djagoun N. 2002. Ingestion volontaire et digestibilité apparente d'une ration à base de la farine de graines de Mucuna pruriens var. utilis complétée de fourrages chez les lapins. Tropicultura, 20: 165-169.

Adesehinwa AOK. 2007. Utilization of Palm Kernel Cake as a Replacement for Maize in Diets of Growing Pigs: Effects on 
Performance, Serum Metabolites, Nutrient Digestibility and Cost of Feed Conversion. Bulgarian Journal of Agricultural Science, 13: 593-600.

Adesehinwa AOK. 2009. Palm kernel cake supplemented with cassava flour waste as energy source for pigs. Revista Brasileira de Ciencias Agrárias, 4: 479-484.

Adesehinwa AOK, Obi OO, Makanjuola BA, Oluwole OO, Adesina MA. 2011. Growing pigs fed cassava peel based diet supplemented with or without Farmazyme 3000 proenx: Effect on growth, carcass and blood parameters. African Journal of Biotechnology, 10(14): 2791-2796.

Agbabiaka LA, Nkwocha GA, Anukam KU, Beketin TO. 2014. Evaluation of Roselle (Hibiscus sabdariffa Linn) calyx meal as dietary supplement in grower pig production. International Journal of AgriScience, 4(6): 293-300.

Bougouma-Yaméogo VMC, Ouédraogo CL, Weremé-Ndiaye A, Konkobo B. 2005. Influence du niveau énergétique de la ration sur les performances de croissance et d'engraissement du porc local : valeur bouchère et rentabilité économique des rations. Revue Africaine de Santé et de Productions Animales, 3(34) : $206-211$

Braine A. 2008. Le point sur le marché français du lapin en 2007- 2008: une filière en crise. Cuniculture Magazine, 35: $60-67$.

Codjo AB. 2003. Estimation des besoins énergétiques du porc local du Bénin encroissance entre 2 et $22 \mathrm{~kg}$ de poids vif. Tropicultura, 21(2): 56-60.

Dube B, Mulugeta SD, Dzama K. 2011. Evaluating Environmental Effects on Growth Performance and Carcass Characteristics of South African Large White Pigs. J. Hum. Ecol., 36(1): 5767.

Ezekwe AG, Machebe NS, Enemona J. 2011. Performance and cost benefit of substituting palm oils sludge (pos) for maize in diets of grower pigs.
International Journal of Science and Nature, 2(2): 210-214.

Fuglie LJ. 2001. The Multiple Uses of Moringa. CTA, CWS: Dakar, Sénégal ; 103-115.

Gidenne T, Lebas F. 2005. Le comportement alimentaire du lapin. 11èmes Journées de la Recherche Cunicole. Paris, 2930/11/2005, 183- 196. ITAVI Ed., Paris.

Hassan MR, Chakrabarti R. 2009. Use of algae and aquatic macrophytes as feed in small-scale aquaculture: A review. FAO Fisheries and Aquaculture technical paper, 531. FAO, Rome, Italy.

Meffeja F, Dongmo T, Fotso J-M. 2006. Teneur énergétique de la ration et performances de croissance du porc au Cameroun. Cahiers Agricultures, 15 (3) : $301-305$.

Mopaté LY, Kabore-Zoungrana CY. 2005. Commerce et consommation de la viande porcine dans quelques villes de l'Afrique de l'Ouest et du Centre. Communication à l'atelier international « IVè Journées Agro-Sylvo-Pastorales », N'Djaména, Tchad, 07/11 au 13/11/2005.

Mopaté LY. 2008. Dynamique des élevages porcins et amélioration de la production en zones urbaine et périurbaine de la ville de N'Djaména (Tchad). Thèse de Doctorat Unique en Gestion Intégrée des ressources Naturelles, option : Productions Animales, Université Polytechnique de Bobo-Dioulasso (UPB), Burkina-Faso, 245 p.

Ovissipour M, Abedian AM, Motamed ZA, Rasco B, Safari R, Shahiri H. 2009. The effect of enzymatic hydrolysis time and temperature on the properties of protein hydrolysates from the Persian sturgeon (Accipenser persicus) viscera. Food Chem., 115: 238-242.

Patricot S. 2002. Les systèmes d'élevages familiaux de porcs au Nord-Viêtnam. Synthèse bibliographique. DESS Productions animales en régions chaudes. Année Universitaire, 2001 - 2002, CiradEmvt, Montpellier, France, 38 p. + annexes $21 \mathrm{p}$. 
Raja W, Rathaur P, John SA, Ramteke PW. 2012. Azolla-Anabaena association and its significance in supportable agriculture. Hacettepe J. Biol. Chem., 40(1): $1-6$.

Saikia P, Bhar R. 2010. Influence of Kitchen/food waste on growth performance of grower piglets. Veterinary World, 3(1):34-36.

Saxena P. 2011. Optimization techniques for animal diet formulation. www. Gate2Biotech.com, 1(2) 1-5.

Wang JF, Wang M, Lin DG, Jensen BB, Zhu YH. 2006. The effect of source of dietary fiber and starch on ileal and fecal amino acid digestibility in growing pigs. Asian-Aust. J. Anim. Sci. 19(7): 10401046.

Wang JF, Zhu YH, Li DF Jorgensen HJ, Jensen BB. 2004. The influence of different fiber and starch types on nutrient balance and energy metabolism in growing pigs. Asian-Aust. J. Anim. Sci., 17(2): 263-270.

Youssao IKA, Mourot J, Gbanboche AB, Adehan R, Akoutey A, Edenakpo A. 2004.Influence du régime alimentaire sur les performances de croissance et les caractéristiques de la carcasse de porc de race locale au Bénin. Revue Africaine de Santé et de Productions Animales, 2(1): 31 - 36.

Zanfi C, Colombini S, Mason F, Galassi G, Rapetti L, Malagutti L, Crovetto GM, Spanghero M. 2014. Digestibility and metabolic utilization of diets containing whole-ear corn silage and their effects on growth and slaughter traits of heavy pigs. J. Anim. Sci., 92: 211-219.

Zanfi C, Spanghero M. 2012. Digestibility of diets containing whole ear corn silage for heavy pigs. Livestock Science, 145: 287291. 